\title{
COMMUNITY RISK PERCEPIION IN COVID-19: A NEGLECTED ISSUE IN PUBLIC HEALTH
}

\author{
Hamid Safarpour ${ }^{1,2}{ }^{\circ}$, Mohammad Esmaeil Motlagh ${ }^{3}{ }^{\circ}$, Moslem Sarani ${ }^{4,5}{ }^{\infty}$, \\ Davoud Pirani ${ }^{4}$, Mehdi Safari ${ }^{4} \odot$, Meysam Safi-Keykaleh ${ }^{6} \odot$ \\ ${ }^{1}$ Non-Communicable Diseases Research Center, Ilam University of Medical Sciences, Ilam, Iran \\ ${ }^{2}$ Department of Nursing, School of Nursing and Midwifery, Ilam University of Medical Sciences, Ilam, Iran \\ ${ }^{3}$ Department of Pediatrics, Ahvaz Jundishapur University of Medical Sciences, Ahvaz, Iran \\ ${ }^{4}$ Department of Health in Disasters and Emergencies, School of Public Health and Safety, Shahid Beheshti University of Medical Sciences, Tehran, Iran \\ ${ }^{5}$ Health management and Social Development Research Center, Golestan University of Medical Sciences, Gorgan, Iran \\ ${ }^{6}$ Nahavand School of Allied Medical Sciences, Hamadan University of Medical Sciences, Hamadan, Iran
}

KEY WORDS: risk perception, COVID-19, coronaviruses, community health, disaster, infectious disease

Disaster Emerg Med J 2021; 6(4): 210-212

To the Editor,

people's perception of epidemic risk significantly depends on the differences in their awareness level [1]. Risk perception is an important determinant of behavior against risks [1], and depends on factors, including cost-benefit of risk, the role of officials' authorities, socio-economic and political context, ideology, trust, and individual interests and awareness [24]. Monitoring of the people's risk perceptions is part of emergency management in major public health disasters and emergencies [5]. In the initial phase of the outbreak of a disease, the communities' risk perception, especially in an emerging disease where resources for prevention, diagnosis, and treatment are limited and there is a delay in active intervention, is important [6]. Furthermore, the communities' responses are essential in early-stage of all epidemics, because non-pharmacological interventions and prevention are the major options in this stage [7].

Compared to other hazards, such as environmental hazards, it is less well known how people perceive the risks associated with emerging infectious diseases [8] such as COVID-19. In late December 2019, the outbreak of the new coronavirus (COVID-19) began in Wuhan, China, and quickly spread to other parts of the world. However, the high rate of transmission and spread of the virus poses serious challenges to controlling the spread of the disease. To deal with such problems, it is necessary to take non-medical measures such as improving personal protection equipment, imposing travel restrictions, and maintaining a social distancing that to achieve such measures, the will of the people plays an important and decisive role [2]. The efficacy and effectiveness of preventive and control measures depend on the level of participation of people with precautionary measures such as wearing a face mask and hand hygiene, considering the physical distance, and following instructions and health regulations [7]. However, encouraging people to adhere to the recommended precautions is still a health problem. Whether people act voluntarily on health and precautionary principles depends on their risk perception of unhealthy threats. In fact, risk perception is one of the main issues in theories of healthy behaviors, including the health belief model and the protection motivation to protect theory [7].

The results of a study showed that there is a high level of risk perception towards COVID-19 and it was impacted by the demographic characteristics of the population [5]. Furthermore, the results of a study on the factors affecting the risk perception of Iranians in COVID-19 showed that religious-cultural, political, cognitive, social, and emotional factors are effective in the risk perception of Iranians in COVID-19 [3]. The results of this study also showed that beliefs

ADDRESS FOR CORRESPONDENCE:

Meysam Safi-Keykaleh, Nahavand School of Nursing and Midwifery, Hamadan University of Medical Sciences, Hamadan, Iran e-mail: meysam.safi@yahoo.com

This article is available in open access under Creative Common Attribution-Non-Commercial-No Derivatives 4.0 International (CC BY-NC-ND 4.0) license, allowing to download articles and share them with others as long as they credit the authors and the publisher, but without permission to change them in any way or use them commercially. 
and culture had the most positive correlation and emotions had the most negative correlation with Iranians' risk perception of the COVID-19 disease [3]. In addition, The new study found that risk perception of COVID-19 is consistently associated with a number of experiential, social, and cultural factors across countries [8]. Despite the variety of cultures, individualistic worldviews, personal experiences, social values, and social reinforcement through friends and family were important determinants of risk perception. Risk perception is significantly associated with the acceptance of health prevention behaviors [8].

Until November 28, 2021, the total number of COVID-19 patients in Iran reached 6108882 and the total number of deaths from this disease reached 129 629. Furthermore, until November 28, 2021, a total of 57408046 people received the first dose of the COVID-19 vaccine. Also, 46510395 people have received the second dose of the vaccine. In addition, 967931 people received the third dose of the vaccine. Taking into account this numbers, the total dose of vaccines injected in Iran reached 104886372 [9].

To control epidemics such as COVID-19, people's participation in preventive measures is critical. People's participation depends on their perception of the risk of the disease and is achieved by raising awareness, sensitizing, and providing trust in them [2]. Trust is one of the effective factors in shaping risk perception [2]. Trust in government's protocols, news, and safety measures against hazards, lead to increased community preparedness [2, 10, 11]. In contrast, a lack of trust in the authorities' warnings leads to a lack of attention to prevention protocols and so a reduction in social activities [2]. To increase people's risk perception of epidemics, it is necessary to take effective measures, given the cultural context of society. Successful risk management of epidemics such as COVID-19 requires a comprehensive perception of the risk and its elements. Increasing real perception of potential risks needs to identify effective ways to influence people, and thus change their attitudes and performance. As changes in the attitude and performance need to change in knowledge, training and exercise are the most important prerequisites for proper risk perception. Different groups of society need different methods and tools to train and risk perception [1], the need for training in this matter is quite felt. Media information sources, including community media platforms and community workers, may increase public perception of risk. It is also useful to assess the psychological and behavioral status of the community to be aware of subsequent interventions and risk communication strategies as the epidemic progresses. Therefore, risk assessment can be an essential step in managing disease epidemic risk. Since a correct and timely assessment of community risk perception can help to effective risk communication management, risk assessment should be performed immediately to reduce the impact of all epidemics, including COVID-19. What is clear is that the community risk perception in COVID-19 is a neglected issue in communicable disease prevention. Not only the knowledge of people's perceptions of risk, but also the empirical, social, cultural, and political factors and their role in creating preventive health behaviors resulting from risk perception of COVID-19 are need to develop, and implement risk-based communication strategies.

\section{Acknowledgements}

we would like to thank all those people who have helped in the writing of this study.

\section{Authors' contributions}

all authors contributed equally. All authors read and approved the final manuscript.

\section{Conflict of interest}

the authors have declared that no competing interests exist.

\section{REFERENCES}

1. Safi-Keykaleh M, Zavareh DK, Safarpour H, et al. Different perception, different behavior, necessity of consensus over "risk" term. Health in Emergencies and Disasters Quarterly. 2018; 3(3): 121-122, doi: 10.29252/nrip.hdq.3.3.121.

2. Khosravi M. Perceived risk of COVID-19 pandemic: the role of public worry and trust. Electron J Gen Med. 2020; 17(4): em203, doi: 10.29333/ejgm/7856.

3. Samadipour E, Ghardashi F. Factors influencing Iranians' risk perception of COVID-19. J Mil Med. 2020; 22(2): 122-129.

4. Wachinger $G$, Renn 0 , Begg $C$, et al. The risk perception paradox - implications for governance and communication of natural hazards. Risk Anal. 2013; 33(6): 1049-1065, doi: 10.1111/j.15396924.2012.01942.x, indexed in Pubmed: 23278120.

5. He $S$, Chen $S$, Kong L, et al. Analysis of risk perceptions and related factors concerning COVID-19 epidemic in Chongqing, China. J Community Health. 2021; 46(2): 278-285, doi: 10.1007/s10900-020-00870-4, indexed in Pubmed: 32592160. 
6. World Health Organization Outbreak Communication Planning Guide. Geneva, 2008.

7. Kwok KO, Li KK, Chan HH, et al. Community responses during early phase of COVID-19 epidemic, Hong Kong. Emerg Infect Dis. 2020; 26(7): 15751579, doi: 10.3201/eid2607.200500, indexed in Pubmed: 32298227.

8. Dryhurst S, Schneider C, Kerr J, et al. Risk perceptions of COVID-19 around the world. Journal of Risk Research. 2020; 23(7-8): 994-1006, doi: 10.1080/13669877.2020.1758193.
9. Iran's Ministry of Health and Medical Education. 2021. https://behdasht.gov.ir/ (2021-11-28).

10. Bradford RA, O'Sullivan JJ, Craats IM, et al. Risk perception - issues for flood management in Europe. Nat Hazards Earth Syst Sci. 2012; 12(7): 2299-2309, doi: 10.5194/nhess-12-2299-2012.

11. Cori L, Bianchi F, Cadum E, et al. Risk perception and COVID-19. Int J Environ Res Public Health. 2020; 17(9): 3114, doi: 10.3390/ ijerph17093114, indexed in Pubmed: 32365710. 